\title{
Shape-dependent orientation of thermophoretic forces in microsystems
}

\author{
Qi Li, ${ }^{1}$ Tengfei Liang, ${ }^{1}$ and Wenjing $\mathrm{Ye}^{1,2, *}$ \\ ${ }^{1}$ Department of Mechanical Engineering, The Hong Kong University of Science and Technology, Clearwater Bay, Kowloon, Hong Kong \\ ${ }^{2}$ KAUST-HKUST Micro/Nanofluidic Joint Laboratory, The Hong Kong University of Science and Technology, \\ Clearwater Bay, Kowloon, Hong Kong
}

(Received 2 June 2013; published 24 September 2013)

\begin{abstract}
It is generally acknowledged that the direction of the thermophoretic force acting on microparticles is largely determined by the imposed temperature gradient, and the shape of the microparticle has little influence on its direction. We show that one type of thermophoretic force, emerged due to the advent of microfabrication techniques, is highly sensitive to object shape, and it is feasible to tune force orientation via proper shape design. We reveal the underlying mechanism by an asymptotic analysis of the Boltzmann equation and point out the reason why the classical thermophoretic force is insensitive to the particle shape, but the force in microsystems is. The discovered phenomenon could find its applications in methods for microparticle manipulation and separation.
\end{abstract}

DOI: 10.1103/PhysRevE.88.033020

PACS number(s): 47.61.Fg, 05.60.Cd, 47.45.Ab, 47.61.Cb

\section{INTRODUCTION}

The seminal observation by Tyndall [1] has led to the discovery of thermophoretic force, a mechanical force acting on a particle in a gas possessing a temperature gradient. The resulting phenomenon, that is, thermophoresis, is the driving force behind many successful practical applications, particularly in the field of aerosol $[2,3]$. The fundamental mechanism of thermophoresis has been extensively researched [4-8]. Unlike thermophoresis in liquids in which particles may move to the hot or the cold side, it is generally acknowledged that for gas thermophoresis the direction of thermophoretic force is always opposite to the temperature gradient and particles migrate from the hot region to the cold region. Nevertheless, the possibility of a reversed force direction was pointed out by Dwyer [9] and further studied by Takata et al. in the nearcontinuum regime [8]. Theoretical studies indicate that when the thermal conductivity of the particle is much larger than that of gas, the direction of thermophoretic force could be along the temperature gradient and negative thermophoresis would take place. Almost all the theoretical and numerical studies assume a spherical shape of the particle. Very little work has been done on the study of the shape effect on thermophoresis, although some work has pointed out that for spheroids in the near-continuum regime the magnitude of the thermophoretic force changes greatly compared to that of the spherical particle [10]. Indeed, the success of numerous applications of thermophoresis based on the assumption that all particles migrate towards the cold region suggests that particle shape plays a negligible role in the direction of thermophoretic force.

With the advent of microfabrication technology, microstructures and nanostructures with integrated heaters can be routinely fabricated, which gives rise to a different type of thermophoretic force, entitled Knudsen force. Similar to the classical thermophoretic force acting on particles, Knudsen force also results from a nonuniform temperature field surrounding the microstructures and nanostructures. In particular, Knudsen force often refers to the force acting on an object that is of uniform temperature but placed in a nonisothermal gas

*Corresponding author: mewye@ust.hk medium, while the classical thermophoretic force refers to the force acting on a particle whose temperature is determined by the imposed field. Measurements of Knudsen forces acting on a heated microbeam embedded in a cold enclosure or next to a cold substrate were reported in Refs. [11-13]. The impact of these forces on promising applications of microsystems cannot be understated and studies have begun to surge in recent years [14-18]. It has been found numerically via the direct simulation Monte Carlo (DSMC) method that in the highKnudsen regime, the orientation of the Knudsen force acting on a rectangular beam in a rectangular enclosure depends on the Knudsen number $(\mathrm{Kn})$ as well as on the shape of the system $[15,17]$. While the fundamental mechanism of the reversibility of force orientation was not revealed in these numerical studies, the findings indicate the importance of the shape of the structures and particles on the Knudsen force, which would have a profound impact on practical applications. In this article, a theoretical study based on the asymptotic theory of the Boltzmann equation is conducted to probe the fundamentals of force reversibility and to investigate the shape effect on thermophoretic forces, including both the classical thermophoretic force and Knudsen force acting on the heated microbeam. The focus of the study is placed on the near-continuum regime in which most applications takes place.

\section{ASYMPTOTIC THEORY}

In this article, two two-dimension model problems, namely, a microparticle immersed in a gas with a temperature gradient and a heated microbeam placed inside a cold chamber, are studied. Our aim is to investigate the mechanism and shape effect on thermophoretic forces acting on the beam and the particle. Of particular interest is the orientation of the force. The schematics of the problem domains are illustrated in Fig. 1, where the corners of the beam and the particle are rounded to create a smooth boundary. The sharpness of the corner can be tuned via its radius. For the microbeam example, the height, the width, and the radius of the corners of the beam and the chamber are denoted by $t L_{0}, l L_{0}, r L_{0}$ and $L_{2} L_{0}, L_{1} L_{0}, R L_{0}$, respectively, with $L_{0}$ being the reference length. The gaps between the beam and the bottom, the top, and the side wall of the chamber are 


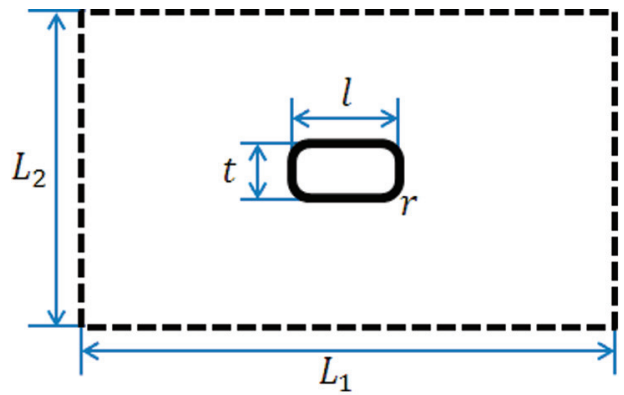

(a)

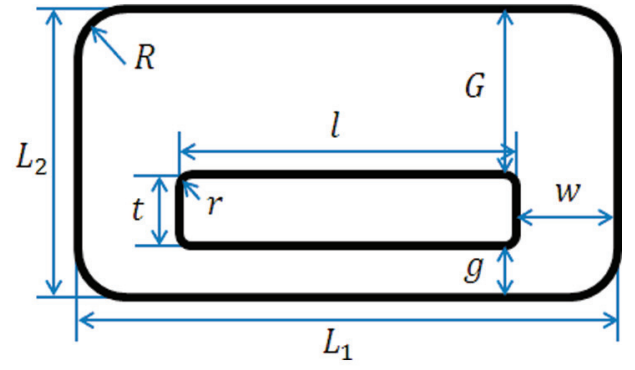

(b)

FIG. 1. (Color online) Schematic illustration of the model problems. (a) Classic thermophoresis; (b) heated microbeam.

denoted by $g L_{0}, G L_{0}$, and $w L_{0}$, respectively. Both the beam and the chamber are isothermal but of different temperatures. As for the thermophoresis problem, the dashed line in the figure indicates the artificial boundary created to set up the temperature gradient and to serve as the boundary for flow analysis. The temperature of the particle is determined by the imposed far-field gas temperature gradient and the particle's thermal conductivity, which is set to be a variable for the investigation of its influence on the force.

The asymptotic theory of the Boltzmann equation is applied to analyze the near-continuum gas behavior in the two problems. Compared with numerical methods that simulate the physical process directly, such as molecular dynamics (MD) and DSMC, this approach is not only much more efficient but also allows probing the fundamental mechanism explicitly. By expanding the velocity distribution function in terms of a power series of the $\mathrm{Kn}$, a set of macroscopic governing equations and the corresponding boundary conditions of various orders can be derived from the Boltzmann equation combined with a fully diffuse gas-wall interaction model using the perturbation technique [8,19]. Specifically, the distribution function and macroscopic variables are expanded as power series of $k$, where $k=\frac{\sqrt{\pi}}{2} \mathrm{Kn}$. Assuming the system is slightly away from its local equilibrium and denoting the nondimensional, nonequilibrium parts of the variables by $P_{i j}, u_{i}, \tau$, and $\omega$ for pressure, velocity, temperature, and density, respectively, the corresponding macroscopic variables are expressed as $P_{0}\left(\delta_{i j}+P_{i j}\right), \quad\left(2 R T_{0}\right)^{1 / 2} u_{i}$, $T_{0}(1+\tau)$, and $P_{0}\left(R T_{0}\right)^{-1}(1+\omega)$, with $P_{0}$ and $T_{0}$ being the reference variables and $R$ being the specific gas constant. The macroscopic governing equations describing the gas behavior outside the Knudsen layer can be derived from the linearized Boltzmann equation and are listed as follows:

$$
\begin{gathered}
\frac{\partial P_{G 0}}{\partial x_{i}}=0, \\
\frac{\partial u_{i G m}}{\partial x_{i}}=0, \\
\frac{\partial P_{G m+1}}{\partial x_{i}}=\gamma_{1} \frac{\partial^{2} u_{i G m}}{\partial x_{j}^{2}}, \\
\frac{\partial^{2} \tau_{G m}}{\partial x_{i}^{2}}=0,
\end{gathered}
$$

where $\gamma_{1}$ is a constant coefficient determined by the molecular model, the subscript $G$ denotes the Grad-Hilbert part, and $m$ indicates the order of macroscopic quantities, that is, $O\left(k^{m}\right)$, in the gas domain. Similarly the boundary conditions of the perturbed quantities at different orders can be formulated. For the model problems considered in this article, the governing equations, together with the corresponding boundary conditions, are summarized in Table I, where $x_{i}$ is the nondimensional spatial variable; $\mathbf{n}$ is the outward normal vector of the solid boundary, and $\mathbf{t}$ is the tangential vector that rotates $90^{\circ}$ from n counterclockwise; $\psi_{G}$ is the stream function, $\frac{\partial \psi_{G}}{\partial x_{1}}=-u_{2}, \frac{\partial \psi_{G}}{\partial x_{2}}=u_{1} ; \bar{\kappa}$ is the curvature of the boundary; $\kappa$ is the thermal conductivity; and $\gamma_{1}, K_{1}, k_{0}, a_{4}, a_{5}, b_{2}$ are constant coefficients determined by the molecular model and gas-surface interaction model.

The requirement of momentum conservation demands that $\frac{\partial P_{i j}}{\partial x_{j}}=0$ and $\frac{\partial P_{i j G}}{\partial x_{j}}=0$. Hence the force acting on the object immersed in the gas can be obtained by simply integrating stresses over any closed path $c$ as long as it encloses the object. In addition, the higher-order derivatives of flow velocity and the integration of thermal stress over a closed curve vanish [19], thus only two terms remain and contribute to the force as shown in Eq. (5):

$$
\begin{aligned}
F_{i}= & -k^{2}\left[\oint_{c}\left(P_{G 2} \delta_{i j}+\gamma_{1} S_{i j G 1}\right) n_{j} d s\right] P_{0} L_{0} \\
& -k^{3}\left[\oint_{c}\left(P_{G 3} \delta_{i j}+\gamma_{1} S_{i j G 2}\right) n_{j} d s\right] P_{0} L_{0},
\end{aligned}
$$

where $S_{i j G m}=-\left(\frac{\partial u_{i G m}}{\partial x_{j}}+\frac{\partial u_{j G m}}{\partial x_{i}}\right)$ and $\mathbf{n}$ is the outward normal vector of $c$. As indicated clearly in Eqs. (T2) and (T4), both $P_{G 2}$ and $S_{i j G 1}$ are resulting from the flow velocity $u_{i G 1}$, which is induced by the tangential temperature gradient at the wall, $\frac{\partial \tau_{G 0}}{\partial t}$. Such a type of flow is called thermal creep flow [20,21]. Another type of thermally induced flow is thermal stress slip flow that is caused by the nonuniform normal temperature gradient along the surface [20,21], as shown in Eqs. (T3) and (T7). The resulting pressure $P_{G 3}$ and shear stress $S_{i j G 2}$ contribute to the second term of the thermophoretic force. Hence the dimensionless force can be expressed as $F / P_{0} L_{0}=F_{\text {creep }} k^{2}+F_{\text {stress }} k^{3}$.

The governing equations are solved numerically using various methods. Specifically, Eqs. (T1) and (T6) are solved by the finite element method (COMSOL) and the boundary element method (BEM), respectively. The BEM is also applied to solve Eqs. (T2), (T3), and (T7) successively to find the flow field. Pressure is then calculated based on Eqs. (T4), (T5), and (T8) using the finite difference method. To validate the accuracy of 
TABLE I. Governing equations and boundary conditions for model problems.

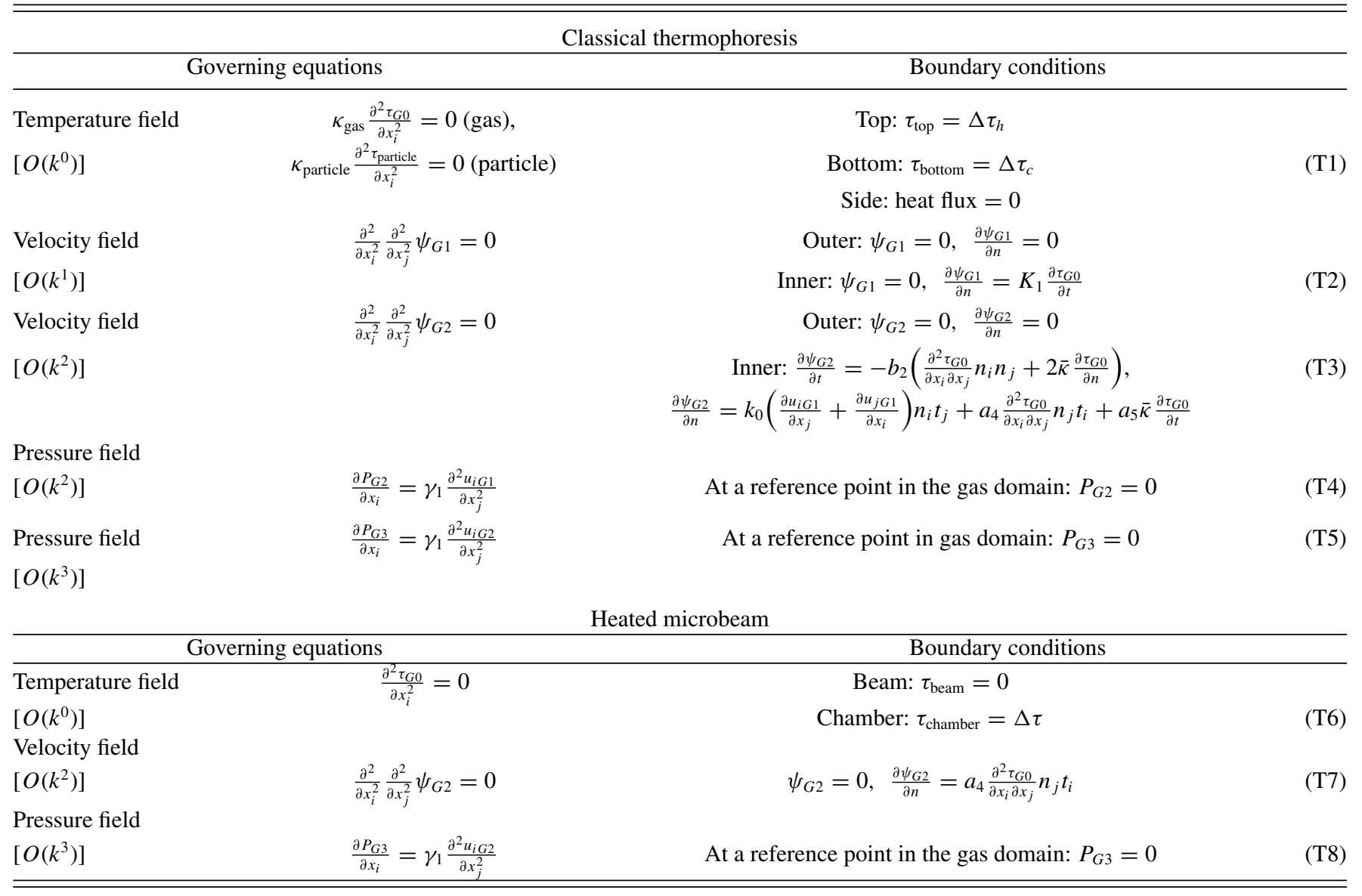

the numerical implementation, the case with two noncoaxial circular cylinders is simulated and numerical results are compared with the available analytical solutions [19].The radiuses of the inner and outer cylinders are $L_{0}$ and $2 L_{0}$, respectively, with a distance $0.5 L_{0}$ between the two centers, and the temperature of the outer cylinder is $10 \%$ lower than that of the inner cylinder. The calculated dimensionless thermophoretic force acting on the inner cylinder is $0.42097 k^{3}$, which is $0.01 \%$ smaller than the analytical value $0.42100 k^{3}$.

\section{RESULTS AND DISCUSSION}

The asymptotic theory of the Boltzmann equation indicates that for a nonisothermal system that is slightly nonequilibrium and with a smooth boundary, thermophoretic force is induced by two types of flow: thermal creep flow and thermal stress slip flow. For the beam example, due to the uniform temperature of the beam, thermal creep flow is not induced in gas and Knudsen force is caused solely by thermal stress slip flow. On the other hand, both thermal creep flow and thermal stress slip flow, which are of the first order and second order of $\mathrm{Kn}$ respectively, are present in the thermophoresis problem and contribute to the thermophoretic force.

\section{A. Classical thermophoresis}

To impose the far-field temperature gradient, a square virtual boundary with dimensions of $100 \times 100$ is employed for a temperature analysis. A similar virtual boundary with dimensions of $500 \times 500$ is used in the velocity simulation to serve as the boundary for the induced gas flow. These dimensions are set so that the virtual boundaries do not disrupt the near-field temperature and velocity around the particle. The dimensionless thickness of the particle $t$ is fixed at 10 while the length $l$ is varied to model different shapes of the particle. The global $\mathrm{Kn}$ is defined based on the thickness of particle $t$. The thermal conductivity ratio of the particle and gas $\kappa_{p} / \kappa_{g}$ ranges from 5 to 1000 .

Due to the finite thermal conductivity ratio, gas temperature and its gradient vary along the surface of the particle. Hence both thermal creep flow and thermal stress slip flow, which are of opposite directions, are induced near the surface of the particle, as shown in Fig. 2, consequently resulting in $F_{\text {creep }}$ and $F_{\text {stress }}$, respectively. Figure 3(a) plots the corresponding $F_{\text {creep }}$ and $F_{\text {stress }}$ defined in the previous section acting on per unit perimeter of the beam in three cases with an aspect ratio $l / t$ varying from 0.5 to 2 . The direction of thermal creep flow is along the thermal gradient, and hence $F_{\text {creep }}$ always pushes the particle towards the cold region. The force due to thermal stress slip flow $F_{\text {stress }}$, on the other hand, pushes the particle to the hot region. The competition between the two forces determines the orientation of the net force. Since the force due to creep flow is $F_{\text {creep }} k^{2}$ and the force due to stress slip flow is $F_{\text {stress }} k^{3}$, when $\mathrm{Kn}$ is very small and $\kappa_{p} / \kappa_{g}$ is not too large, thermal creep flow dominates and hence the net force is opposite to the direction of the temperature gradient, which is observed in most experiments. As $\kappa_{p} / \kappa_{g}$ increases, both forces decrease. 


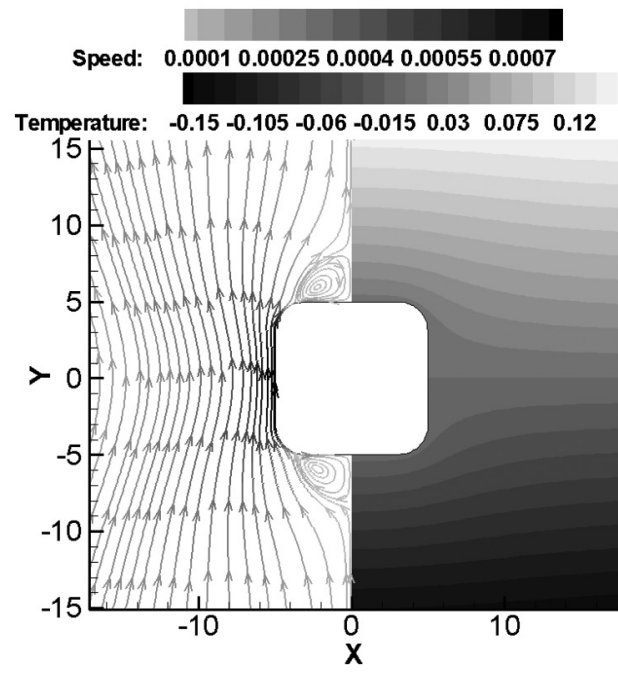

(a)

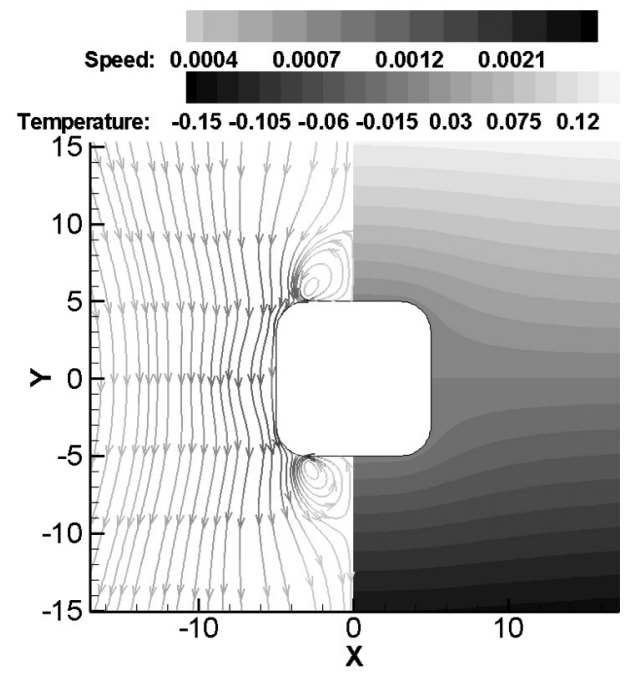

(b)

FIG. 2. Flow structure shown in the left-half domain and temperature contour $\left[O\left(k^{0}\right)\right]$ shown in the right-half domain of the classical thermophoresis particle: (a) flow field of $\left[O\left(k^{1}\right)\right]$; (b) flow field of $\left[O\left(k^{2}\right)\right]$.

However, $F_{\text {creep }}$ approaches zero while $F_{\text {stress }}$ approaches a finite value in the limiting case. Therefore there exists a point at which thermophoretic force reverses its direction and negative thermophoresis takes place. An interesting fact is that the point at which negative thermophoresis occurs can be tuned by particle shape, as shown in Fig. 3(b). Both the aspect ratio and the sharpness of the corners affect the force direction. From the temperature contour shown in Fig. 2, it is clear that thermal creep flow is mainly induced along the thickness of the particle [Fig. 2(a)], and hence a large aspect ratio $l / t$ would weaken the creep flow and thus favor negative thermophoresis. Thermal stress slip flow, on the other hand, is caused by the changing normal temperature gradient which concentrates mostly near the corners of the particle [Fig. 2(b)]. A sharp corner which enhances the stress slip flow would therefore favor negative thermophoresis. However, the tunable range is very small and the force direction is dictated mostly by the imposed temperature gradient.

\section{B. Heated microbeam}

The dimensionless Knudsen force acting on the microbeam is calculated for the case with the following parameters: $t=2$, $l=10, r=0.4, L_{2}=8, L_{1}=20, R=1.0$, and temperature $\tau_{\text {beam }}=0, \tau_{\text {chamber }}=\Delta \tau=-0.1$. The global $\mathrm{Kn}$ is defined based on the bottom gap $g$. The magnitude of the force is $0.6603 k^{3}$ and its direction points downwards, that is, towards the narrow gap. As a comparison, a topographically equivalent system consisting of two noncoaxial circular cylinders with the same temperature conditions is considered, and the calculated

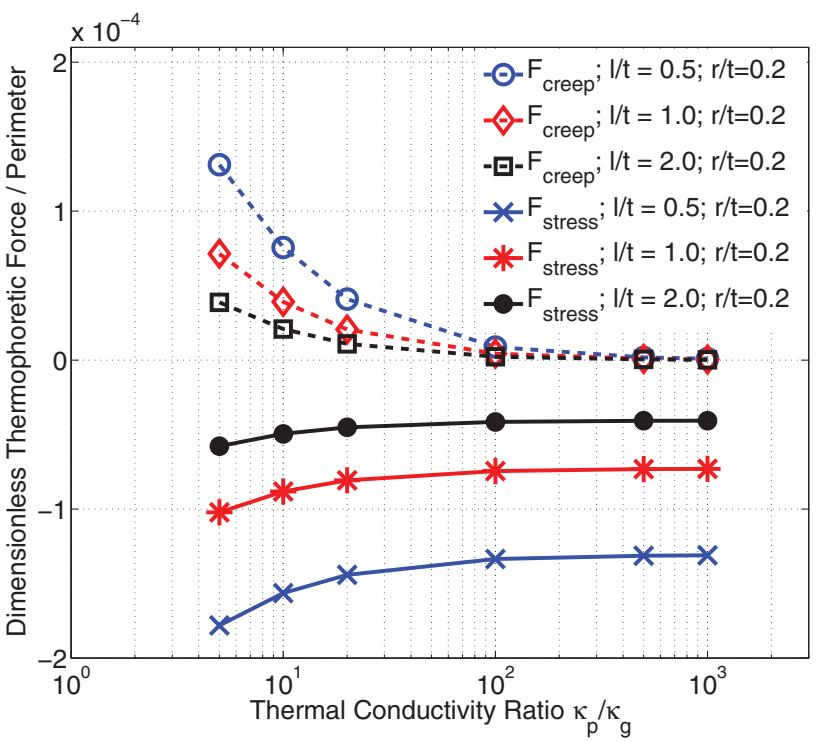

(a)

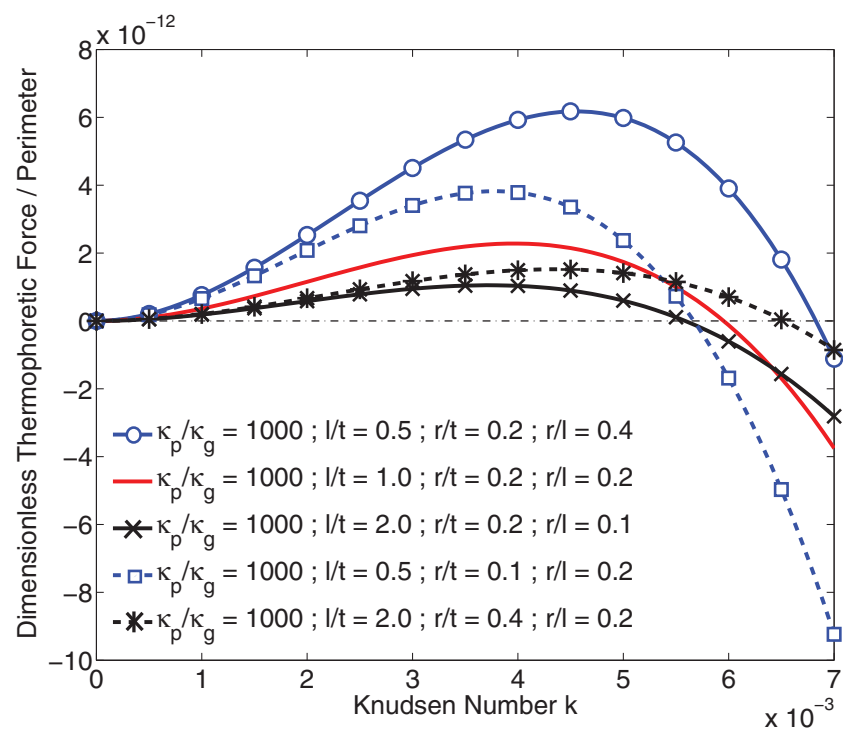

(b)

FIG. 3. (Color online) Shape effect on the thermophoretic force. 


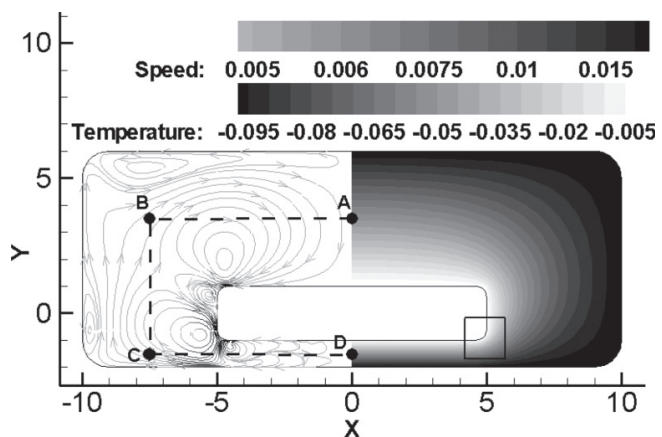

(a)

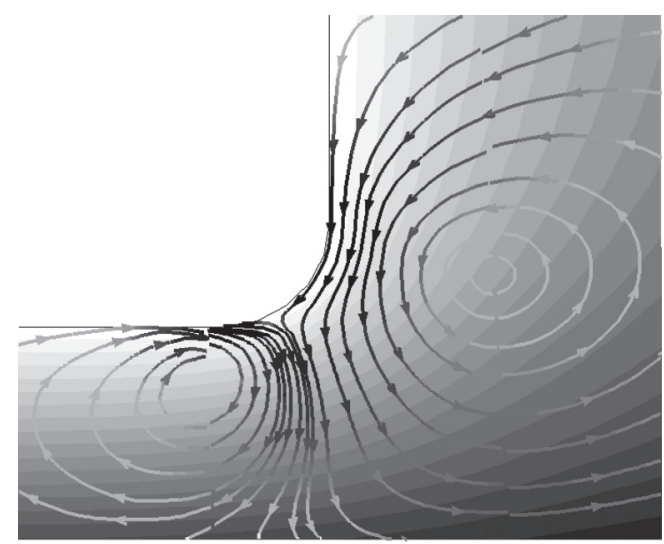

(b)

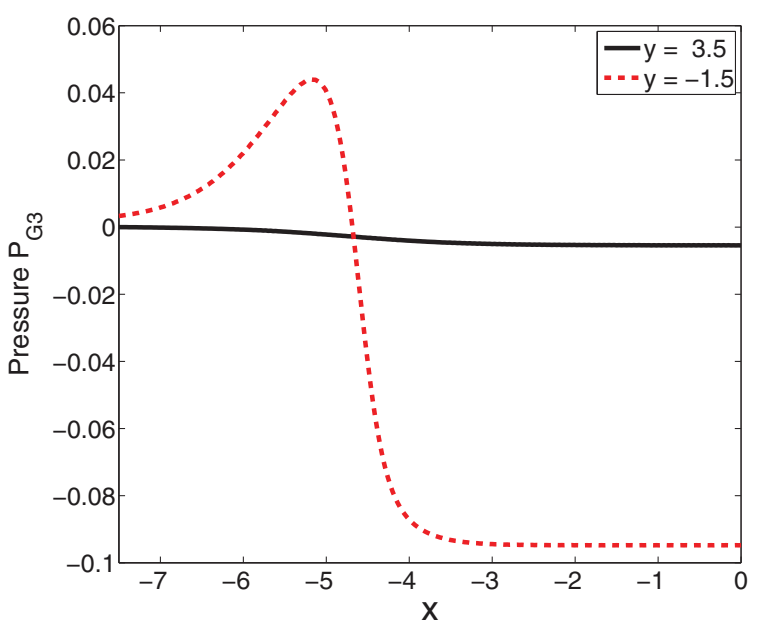

(c)

FIG. 4. (Color online) Flow structure, temperature contour, and pressure variation of gas surrounding a rectangular microbeam; (a) flow structure (left-half domain) and temperature distribution (right-half domain); (b) a magnified subplot of the flow field near the lower right corner; (c) pressure variation along two lines BA $(y=3.5)$ and $\operatorname{CD}(y=-1.5)$.

Knudsen force acting on the inner cylinder has a magnitude of $0.4209 k^{3}$, but points upwards. The opposite force directions in the two systems can be understood by examining the temperature contours, flow structures, and pressure variations plotted in Fig. 4. Along the lower right corner of the microbeam, as indicated in the magnified subplot [Fig. 4(b)], the nonmonotonically varied normal temperature gradient near the corner of the microbeam induces thermal stress slip flows that flow from the

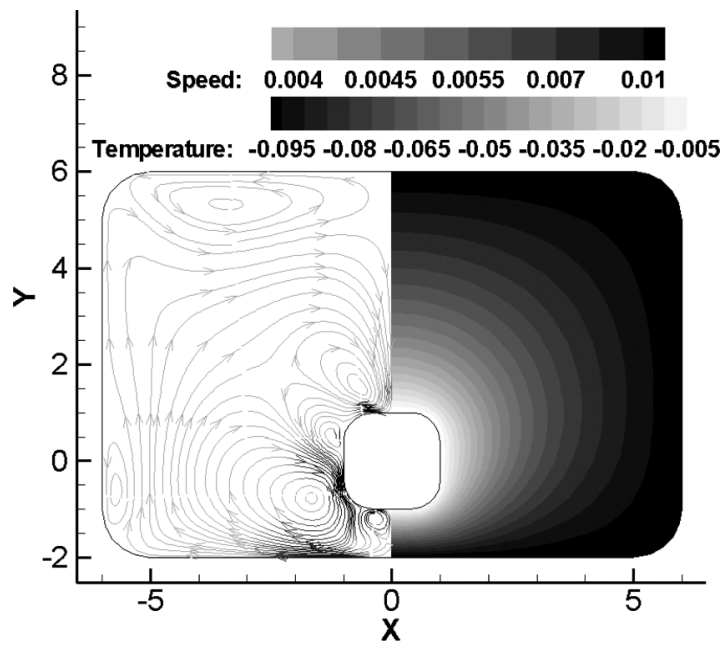

FIG. 5. Flow structure (left-half domain) and temperature contour (right-half domain) of gas surrounding a microbeam with a square cross section with rounded corners.

two sides towards the corner and form two vortexes of opposite directions. While the counterclockwise vortex trends to pump the gas into the narrow gap near the corner, it is blocked by the clockwise vortex which pumps gas out of the narrow gap. Due to symmetry, the flow structure of the left domain is a mirror image of that of the right domain. The counterclockwise vortex inside the narrow gap pumps gas out of the gap, while the clockwise vortex located near the entrance of the narrow gap tends to pump gas inside the gap. As a result, as shown in Fig. 4(c), from point $C$ to $D$, gas pressure increases through a clockwise vortex first, but decreases sharply due to the counterclockwise vortex, resulting in a large low pressure region underneath the beam. On the top of the beam, the pressure only decreases slightly from the equilibrium value along the line $\mathrm{AB}$ due to the relatively weak clockwise vortex. Hence a downward net force is formed. As the microbeam becomes more round, the decreasing trend of the normal temperature gradient enhances, which causes changes in the relative strength and the coverage of the two vortexes. As a result, the clockwise vortex gets stronger while the counterclockwise vortex becomes weaker, as shown in Fig. 5. In the limiting case, that is, the cylinder case, the normal temperature gradient monotonically decreases around the corner towards the narrow gap. The counterclockwise vortex vanishes and only one giant vortex is formed. This vortex pumps gas into the narrow gap and increases the pressure monotonically from point $\mathrm{A}^{\prime}$ to $\mathrm{B}^{\prime}$ (see Fig. 6), resulting in an upward force. The contribution of the viscous stress $S_{i j G 2}$ to the Knudsen force is much smaller than that of pressure $P_{G 3}$, and hence has little influence on the force orientation.

As implied in the above results, the orientation of the force is largely influenced by the shape of the beam due to its effects on the temperature field. Similarly, the configuration of the system, which also plays an important role in the temperature field, would have a strong influence on the force orientation. To further investigate its effects, five independent parameters, namely, $r / t, l / t, G / g, w / g$, and $t / g$, are constructed. Among these parameters, $r / t$ and $l / t$ dictate the shape of the beam, $G / g$ and $w / g$ determine the location of the beam with respect 


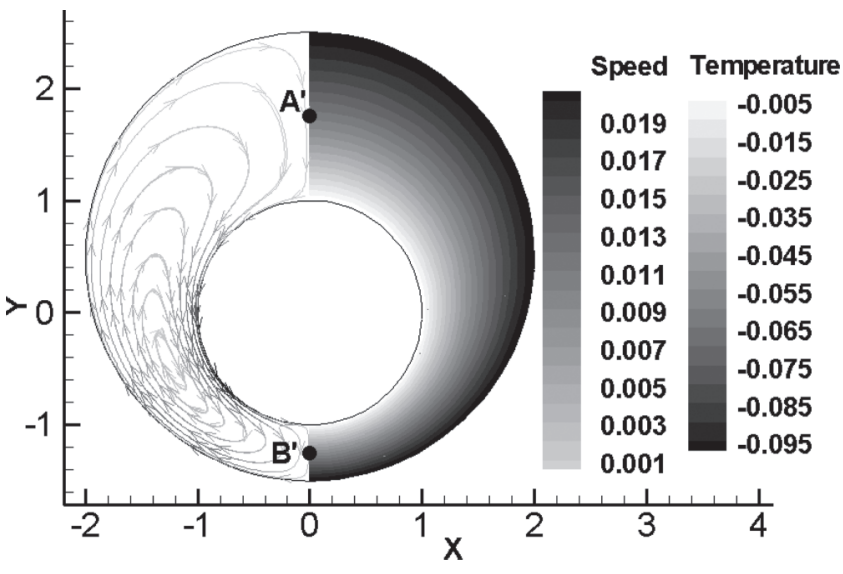

(a)

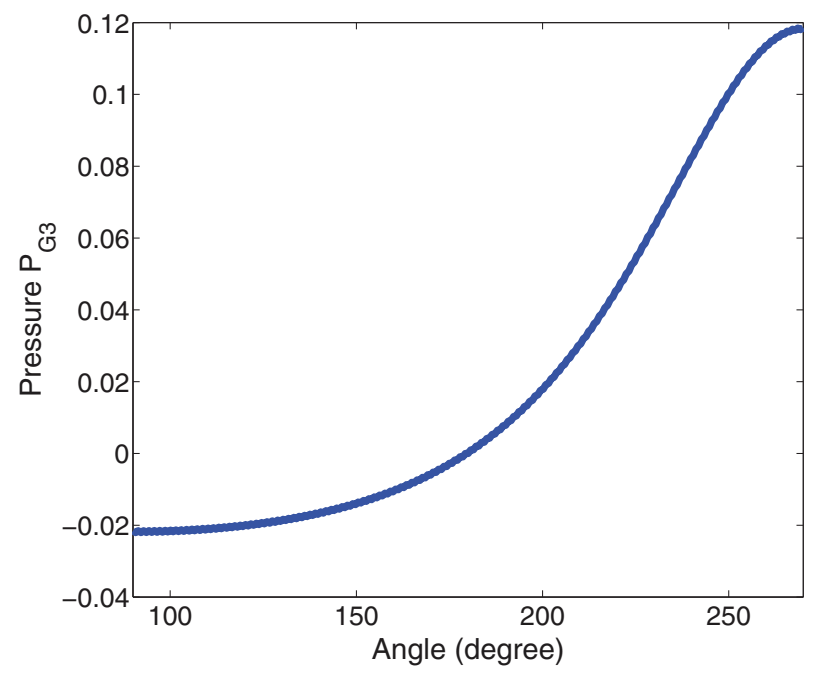

(b)

FIG. 6. (Color online) Flow structure, temperature contour, and pressure variation of gas surrounding a cylindrical microbeam; (a) flow structure (left-half domain) and temperature distribution (right-half domain); (b) pressure variation along the curve $\mathrm{A}^{\prime} \mathrm{B}^{\prime}$ located halfway between the two cylinders.

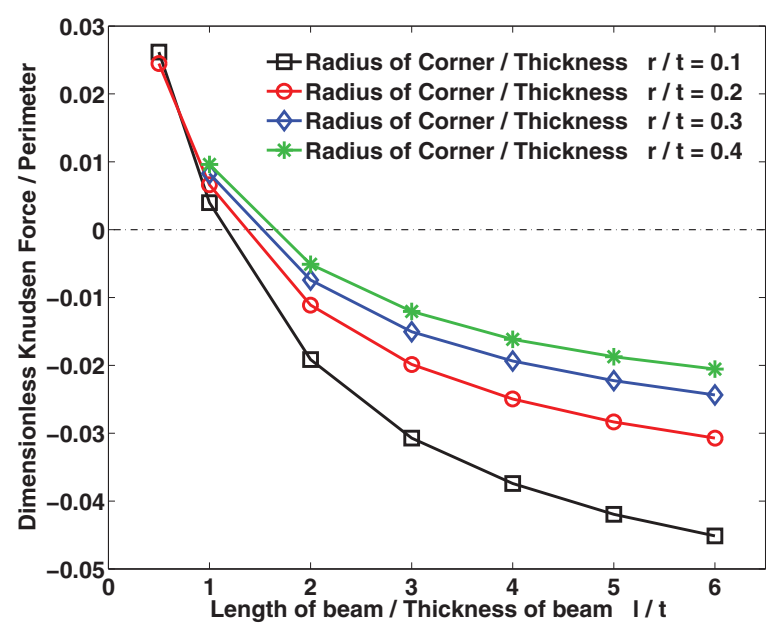

(a)

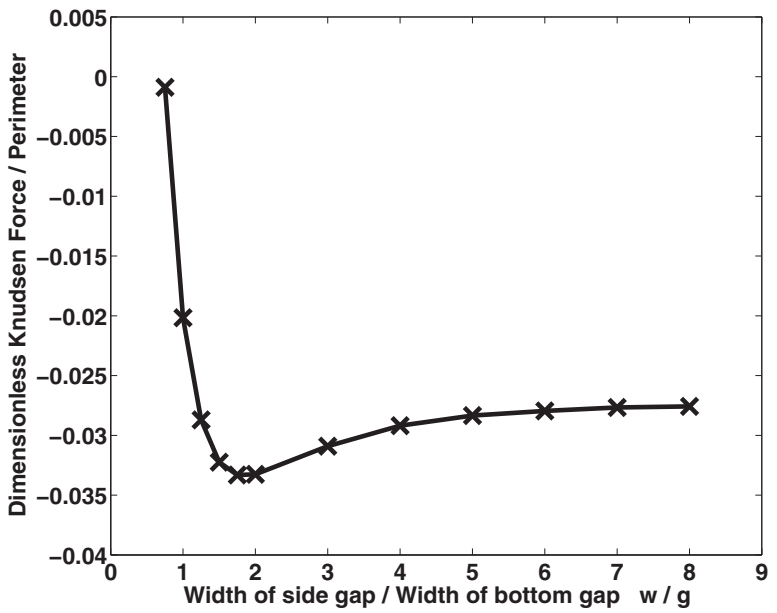

(c)

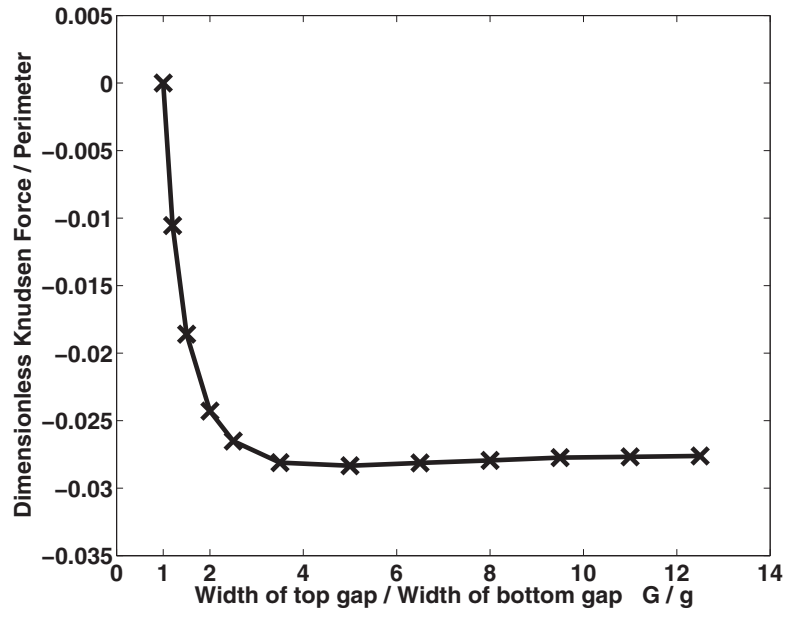

(b)

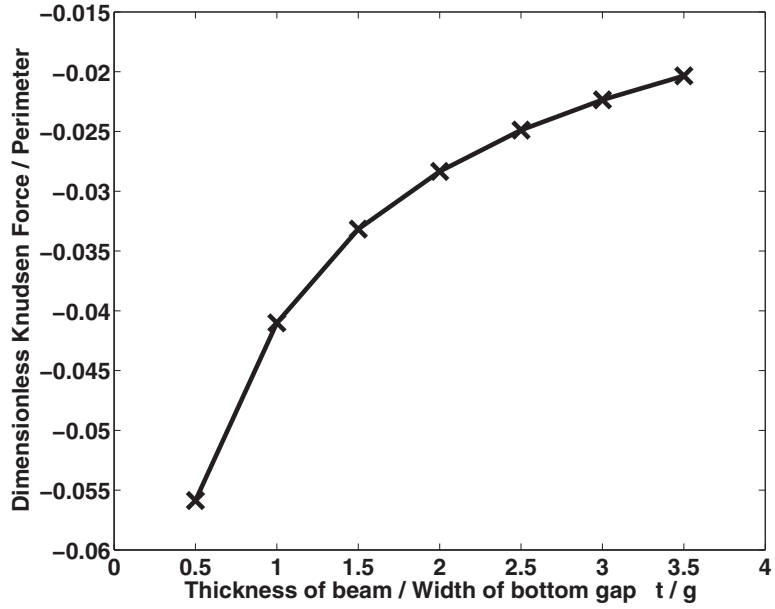

(d)

FIG. 7. (Color online) (a) Knudsen force acting on a unit of the perimeter as a function of the radius of the corner and the aspect ratio of the microbeam; (b) the ratio of the width of the top gap and the width of the bottom gap; (c) the ratio of the width of the side gap and the width of the bottom gap; (d) the relative size of the beam with respect to the chamber. 
to the chamber, and $t / g$ measures the relative size of the beam with respect to the gas domain.

The shape effects of the beam on the force orientation are demonstrated in Fig. 7(a), where Knudsen forces acting on per unit perimeter of the microbeam with varied $r / t$ and $l / t$ are plotted. As mentioned previously, the pressure variation along the beam, and thus the net force, is closely related to the thermally induced vortices which in turn depend on the sharpness of the corners. A sharper corner produces a more rapidly changing normal temperature gradient along the corner, resulting in a stronger counterclockwise vortex underneath the beam. More gas is then pumped out from the narrow gap. Hence beams with sharp corners favor the downward force, as demonstrated in the figure. For a fixed $r / t$, the force orientation changes from the upward direction to the downward direction as the ratio $l / t$ increases. It is interesting to note that there exists a critical value of $l / t$ for each fixed $r / t$ at which the Knudsen force becomes zero.

In additional to the shape of the beam, the location of the beam also has a significant influence on the Knudsen force. Figures 7(b) and 7(c) plot Knudsen force as a function of $G / g$ and $w / g$, respectively. As the ratio $G / g$ increases, the difference between the temperature gradients near the top and the bottom of the beam increases. As such the pressure difference also enlarges, resulting in a force with an increasing magnitude. However, when the top gap $G$ increases to a certain level, the temperature contour near the top of the beam is no longer affected by the chamber wall, and the force eventually approaches a constant value. The dependence of force on the ratio $w / g$ is somewhat different. With increased $w / g$, the magnitude of the force increases first and then decreases before reaching a constant value, as shown in Fig. 7(c). To understand this trend, the evolution of the flow pattern near the lower left corner of the beam is examined and shown in Fig. 8. It is evident that the vortex structure near the corners of both the beam and the chamber is very sensitive to the ratio $w / g$, and the flow induced near the chamber also influences the pressure significantly, particularly when the ratio is small. At $w / g=1$, four vortices are formed, with two of them attached to the chamber and the other two attached to the beam, as illustrated in Fig. 8(a). Both vortices B and D pump gas out of the bottom gap along the walls of the chamber and the beam, respectively, while vortex $\mathrm{C}$ intends to inject gas into the bottom gap, but is blocked by vortex $\mathrm{D}$. As the ratio increases, vortex $B$ starts to merge with vortex $C$, and distributes the gas pumped out from the bottom gap into the side gap, resulting in a lower pressure region underneath the beam and thus a larger downward force. This trend continues as the merged vortex grows larger and stronger until it overpowers vortex D, at which time the merged vortex starts to enter into the bottom gap and injects gas from the side gap into the bottom gap, as shown in Fig. 8(e). The magnitude of the downward force is thus reduced. However, such a decreasing trend eventually saturates because, at a certain point, the vortex structure stabilizes and further increasing $w$ would have no effect on the vortex structure near the corner of the beam. Lastly, the effect of the relative size of the beam with respect to the chamber is shown in Fig. 7(d). A larger beam tends to be subjected to a smaller force per unit perimeter, but a larger net force.

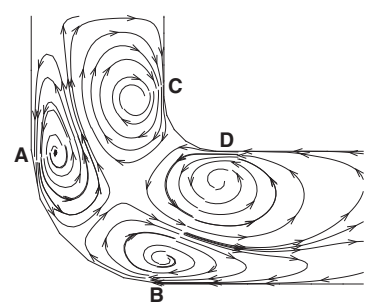

(a)

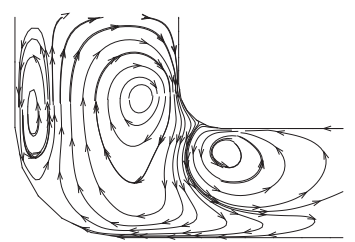

(c)

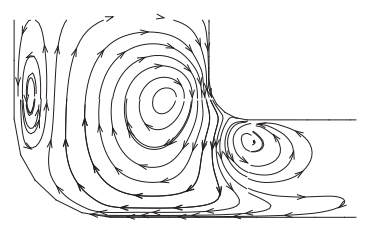

(e)

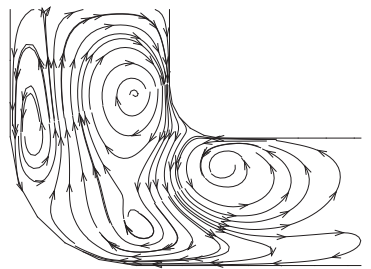

(b)

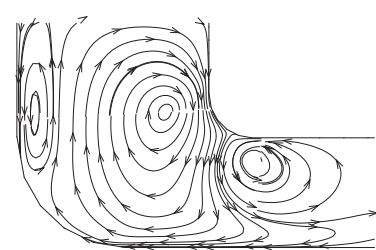

(d)

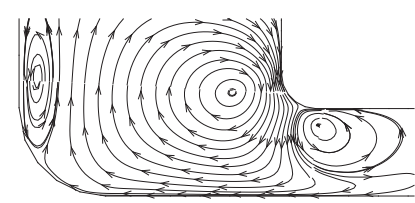

(f)
FIG. 8. Evolution of the flow structure near the lower left corner of the beam; (a) $w / g=1.0$; (b) $w / g=1.25$; (c) $w / g=1.5$; (d) $w / g=1.75$; (e) $w / g=2.0$; (f) $w / g=3.0$.

\section{CONCLUSIONS}

The fundamental mechanism of thermally induced mechanical force in the near-continuum regime is investigated and the influence of object shape on the force orientation is demonstrated in both the classical thermophoresis and heated microbeam cases. The tunable range of classical thermophoretic force using particle shapes is very small as the temperature field around the particle is mostly determined by the imposed far-field temperature gradient. However, in the microbeam case, the force orientation is much more sensitive to the system configuration due to its strong influence on the temperature field, indicating the feasibility of these approaches to manipulating the motion of particles and objects by controlling the gas temperature field via shape design. Another important observation resulting from this study is that for an asymmetric object or an object oriented asymmetrically with respect to the chamber, due to the shape effect, it is possible to have a net torque in addition to the net force produced and acted upon the object. Such a torque may induce a noticeable rotation of the object, which could be utilized to demonstrate negative force and to create a type of rotational engine. This topic is currently under investigation.

\section{ACKNOWLEDGMENTS}

This work is financially supported in part by Award No. SA-C0040/UK-C0016, made by King Abdullah University of Science and Technology, and in part by the Hong Kong Research Grants Council under Competitive Earmarked Research Grant No. 621408. 
[1] J. Tyndall, Proc. R. Inst. 6, 1 (1870).

[2] B. Wang, S. Tao, and D. R. Chen, Aerosol Sci. Technol. 46, 1227 (2012).

[3] O. K. Mohammed Hafiz and A. Singh, Chem. Eng. Res. Des. 89, 593 (2011).

[4] P. S. Epstein, Z. Phys. 54, 537 (1929).

[5] J. R. Brock, J. Colloid Sci. 17, 768 (1962).

[6] Y. Sone, Phys. Fluids 15, 1418 (1972).

[7] Y. Sone and K. Aoki, Phys. Fluids 20, 571 (1977).

[8] S. Takata and Y. Sone, Eur. J. Mech. B/Fluids 14, 487 (1995).

[9] H. A. Dwyer, Phys. Fluids 10, 976 (1967).

[10] M. Williams, J. Phys. D 19, 1631 (1986).

[11] A. Passian, R. J. Warmack, T. L. Ferrell, and T. Thundat, Phys. Rev. Lett. 90, 124503 (2003).

[12] A. Passian, R. J. Warmack, A. Wig, R. H. Farahi, F. Meriaudeau, T. L. Ferrell, and T. Thundat, Ultramicroscopy 97, 401 (2003).
[13] B. Gotsmann and U. Durig, Appl. Phys. Lett. 87, 194102 (2005).

[14] A. Passian, A. Wig, F. Meriaudeau, T. L. Ferrell, and T. Thundat, J. Appl. Phys. 92, 6326 (2002).

[15] T. Zhu and W. Ye, Phys. Rev. E 82, 036308 (2010).

[16] J. Nabeth, S. Chigullapalli, and A. A. Alexeenko, Phys. Rev. E 83, 066306 (2011).

[17] T. Zhu, W. Ye, and J. Zhang, Phys. Rev. E 84, 056316 (2011).

[18] A. A. Donkov, S. Tiwari, T. Liang, S. Hardt, A. Klar, and W. Ye, Phys. Rev. E 84, 016304 (2011).

[19] Y. Sone and S. Tanaka, in Theoretical and Applied Mechanics, edited by F. P. J. Rimrott and B. Tabarrok (North-Holland, Amsterdam, 1980), p. 405.

[20] Y. Sone, in Rarefied Gas Dynamics, edited by D. Dini (Editrice Tecnico Scientifica, Pisa, 1971), Vol. 2, p. 737.

[21] Y. Sone, Annu. Rev. Fluid Mech. 32, 779 (2000). 